\title{
Review
}

\section{The pathology of familial breast cancer How do the functions of BRCA1 and BRCA2 relate to breast tumour pathology? David Bertwistle and Alan Ashworth}

The Institute of Cancer Research, London, UK

Received: 1 September 1999

Revisions requested: 6 September 1999

Revisions received: 16 September 1999

Accepted: 6 October 1999

Published: 27 October 1999

(c) Current Science Ltd

\author{
Important note about how to cite this article \\ This article is also available online in the Breast Cancer Research \\ website. To avoid confusion, please ensure that only the online version \\ of the article is cited in any reference, as follows: \\ Bertwistle D, Ashworth A: The pathology of familial breast cancer: how \\ do the functions of $B R C A 1$ and $B R C A 2$ relate to breast tumour \\ pathology? [review]. http://breast-cancer-research.com/vol1no1/ \\ 27oct99/review/4
}

\begin{abstract}
Women with mutations in the breast cancer susceptibility genes, BRCA1 and BRCA2, have an increased risk of developing breast cancer. Both BRCA1 and BRCA2 are thought to be tumour suppressor genes since the wild type alleles of these genes are lost in tumours from heterozygous carriers. Several functions have been proposed for the proteins encoded by these genes which could explain their roles in tumour suppression. Both BRCA1 and BRCA2 have been suggested to have a role in transcriptional regulation and several potential BRCA1 target genes have been identified. The nature of these genes suggests that loss of BRCA1 could lead to inappropriate proliferation, consistent with the high mitotic grade of BRCA1-associated tumours. BRCA1 and BRCA2 have also been implicated in DNA repair and regulation of centrosome number. Loss of either of these functions would be expected to lead to chromosomal instability, which is observed in BRCA1 and BRCA2associated tumours. Taken together, these studies give an insight into the pathogenesis of $B R C A$-associated tumours and will inform future therapeutic strategies.
\end{abstract}

Keywords: BRCA1, BRCA2, breast cancer, cell cycle checkpoints, DNA repair, gene expression

\section{Introduction}

About one in 12 women in the Western world develop cancer of the breast, and at least $5 \%$ of these cases are thought to result from a hereditary predisposition to the disease $[1,2]$. Two breast cancer susceptibility genes in humans (BRCA1 and BRCA2) have been mapped and cloned, and mutations in these genes account for most families with four or more cases of breast cancer diagnosed before the age of 60 years. (Note that 'Brcal' and 'Brca2' are used in the following discussion to denote the equivalent genes in mice, and that Roman text indicates the encoded protein.) Women who inherit loss-of-function mutations in either of these genes have approximately $85 \%$ risk of developing breast cancer by age 70 years [3]. Both BRCA1 and $B R C A 2$ are thought to be tumour-suppressor genes, because the wild-type allele of the gene is observed to be lost in tumours of heterozygous carriers. As well as breast cancer, carriers of mutations in these genes are at elevated risk of cancer of the ovary, prostate and pancreas. Surprisingly, however, despite the association with inherited predisposition, somatic disease-causing mutations in BRCA1 or $B R C A 2$ are extremely rare in sporadic breast cancers $[1,2]$. 

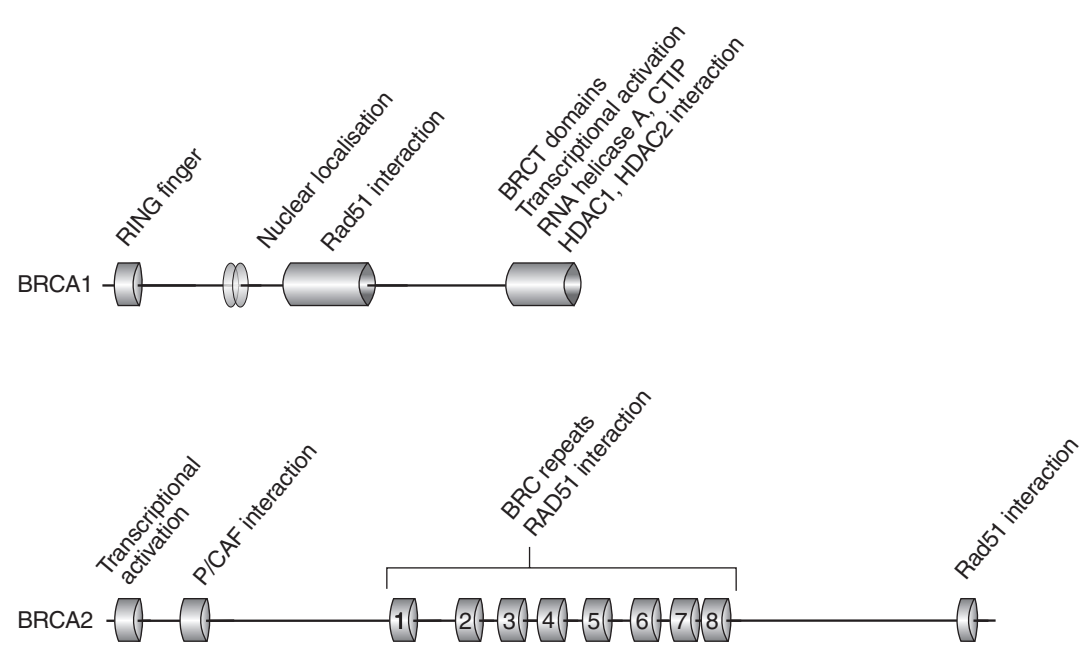

Features of the BRCA1 and BRCA2 proteins. Structures, features and regions of interaction with other proteins discussed in this review are indicated.

The BRCA1 gene is made up of 22 coding exons and encodes a protein of 1863 amino acids [ $4^{\bullet \bullet}$. Most of the coding region shows no sequence similarity to previously described proteins, apart from the presence of a RING zinc finger domain at the N-terminus of the protein and two 'BRCT' repeats at the C-terminus [2] (Fig. 1). The $B R C A 2$ gene has 26 coding exons and encodes a protein of 3418 amino acids, with an estimated molecular weight of $384 \mathrm{kDa}\left[2,5^{\circ}\right]$. The only obvious feature of the BRCA2 protein is the presence of eight copies of a 30-80 amino acid repeat (the BRC repeat) in the part of the protein encoded by exon $11\left[6^{\bullet}, 7^{\circ}\right]$ (Fig. 1). These repeats are able to bind the RAD51 protein implicated in DNA repair and recombination $\left[8^{\bullet}, 9^{\bullet}\right]$.

Functions for the BRCA proteins in both transcriptional regulation and DNA repair/recombination have been suggested [2]. It is still unclear, however, how loss of BRCA gene function leads to tumourigenesis. Important clues to this process are provided by the pathological features of breast tumours in women who are carriers for mutations in BRCA1 or BRCA2. These tumours are clearly distinct from each other, as well as from sporadic tumours $\left[10^{\bullet \bullet}\right]$. This indicates that, despite many similarities in these genes [2], they must have at least some nonredundant functions. The evidence for the proposed functions of the BRCA1 and BRCA2 proteins are reviewed and related to some of the pathological features of $B R C A$-associated tumours.

\section{Transcriptional regulation}

The presence in BRCA1 of a RING finger domain, which are frequently found in transcriptional regulatory proteins, led to the tacit assumption that BRCA1 might be involved in the transcriptional regulation of genes [4*0]. Subsequently, however, it has become clear that RING finger domains are found in a wide variety of proteins of different function, and are indicative of protein-protein interaction rather than interaction with DNA. Nevertheless, there is significant accumulating evidence for a role in transcriptional regulation for BRCA1, and to a lesser extent for BRCA2. Disregulation of target genes consequent to the loss of $B R C A$ genes is a plausible mechanism to explain the pathological features of $B R C A$-associated tumours. As described below, however, the exact function of the BRCA proteins in transcriptional regulation is not yet understood.

A C-terminal fragment of BRCA1, which is fused to the GAL4 DNA-binding domain, activates transcription of reporter genes, suggesting that BRCA1 might regulate transcriptional activation $\left[11^{\bullet}-13^{\bullet}\right]$. The finding that endogenous, full-length BRCA1 copurifies with the RNA Pol II holoenzyme complex via an interaction with RNA helicase A supports such a role for BRCA1 $\left[14^{\bullet}, 15,16\right]$. It is worth noting, however, that RAD51 can also be found in this complex despite having no known role in transcriptional regulation [17].

Several groups have used overexpression of BRCA1 in various cell lines to find targets of BRCA1 transcriptional regulation (Table 1). Most work has concentrated on the cell division kinase (CDK) inhibitor, $\mathrm{p} 21^{\mathrm{WAF} 1}$, which acts to arrest the cell cycle at the $G_{1}$ to $S$ phase and $G_{2}$ to $M$ phase transitions [18]. In COS cells, 293 T cells and human colon cancer cells, reporter constructs containing portions of the $p 21^{W A F 1}$ promoter are activated by overexpression of 
Table 1

\begin{tabular}{lcl}
\multicolumn{2}{l}{ Suggested transcriptional targets of BRCA1 } \\
\hline Gene & Regulation & Function \\
\hline$p 21$ WAF1 & $\uparrow \downarrow^{*}$ & inhibitor of cyclin dependant kinases \\
$B A X$ & $\uparrow$ & proapoptotic \\
GADD45 & $\uparrow$ & G2/M checkpoint \\
MDM2 & $\uparrow$ & regulation of p53 \\
CDC25A & $\downarrow$ & phosphatase/cell cycle regulation \\
Synthetic estogen & $\downarrow$ & \\
receptor responsive promoter & \\
\hline
\end{tabular}

${ }^{*}$ Overexpression of BRCA1 activates $p 21$ WAF1 expression $\left[19^{\circ}, 20-22\right]$. However, p21WAF1 is also activated in BRCA1-null mice [29・*].

BRCA1 [19²0-22]. Furthermore, in colon cancer cells, BRCA1 overexpression leads to an increase in p21 WAF1 protein levels, accompanied by cell cycle arrest [19*]. There may be some cell-type specificity in this, however, because overexpression of BRCA1 in osteosarcoma cells does not induce increased $\mathrm{p} 21^{\mathrm{WAF} 1}$ protein levels [23 $3^{\circ}$. Other studies suggest that BRCA1 may also be able to activate the promoters of the proapoptotic gene $B A X$, the $\mathrm{G}_{2}$ to $\mathrm{M}$ phase checkpoint gene $G A D D 45$, and the p53 regulatory gene $M D M 2\left[20,21,23^{\circ}\right]$.

There are also some indications that BRCA1 may act as a repressor of transcription. BRCA1 overexpression has been shown to inhibit transcription from the promoter of the cell-cycle regulatory gene $C D C 25 A$ and from a synthetic oestrogen receptor-responsive promoter [24,25 $]$. This may involve the interaction of BRCA1, via CtIP, with the transcriptional corepressor $\mathrm{CtBP}\left[26^{\bullet}, 2^{\bullet}\right]$. CtBP can inhibit transcription by binding the histone deactylase HDAC1, which has been found in BRCA1-containing complexes [28 $8^{\circ}$. When BRCA1 alone is overexpressed in 293 cells, a $p 21^{\text {WAF1 }}$ promoter-reporter construct is activated, but when CtIP and CtBP are coexpressed it is not $\left[27^{\circ}\right]$. Interestingly, interaction of BRCA1 with CtIP and $\mathrm{CtBP}$ is disrupted upon DNA damage, suggesting a role for BRCA1 in the DNA damage-dependent induction of p2 $1^{\mathrm{WAF} 1}\left[2^{\circ}\right]$. Because loss of Brca1 in mouse embryos also leads to induction of $\mathrm{p} 21^{\mathrm{WAF} 1}\left[\mathbf{2 9}^{\circ} \cdot\right.$ ], however, the role of $\mathrm{BRCA} 1$ in $\mathrm{p} 21^{\mathrm{WAF} 1}$ regulation is not clear.

There are, as yet, no reports of BRCA1 binding DNA and acting directly as a transcription factor. Rather, BRCA1 appears to exert its influence on transcription as a cofactor or adaptor, because it can interact with both DNA-binding transcription factors and the RNA Pol II holoenzyme. The influence of BRCA1 on the $p 21^{W A F 1}, B A X$ and $M D M 2$ promoters is mediated, at least in part, by p53, with which BRCA1 has been shown to physically interact [20-22].
Transcriptional regulation has also been proposed as a function of BRCA2, because sequences encoded by exon three, when fused to the GAL4 DNA-binding domain, can activate transcription of a reporter gene [30 $0^{\circ}$. It is not clear whether these sequences can function similarly in the context of the intact protein, however. Other evidence supporting a role for BRCA2 in transcriptional regulation is that it can associate with histone acetyltransferase (HAT) activity. The exact nature of this association is not clear, because one study suggested that BRCA2 has intrinsic HAT activity $\left[31^{\circ}\right.$ ], whereas another suggested that it is associated with HAT activity by virtue of an interaction with $\mathrm{P} / \mathrm{CAF}$, which possesses HAT activity [32 $2^{\bullet}$. Association with HAT activity does not necessarily denote a role in transcriptional regulation, however. It could also reflect a role in chromatin remodelling, which might be required for the proposed role of BRCA2 in homologous recombination, which is discussed below.

The promoters that BRCA1 has been shown to activate are those for stress-induced genes involved in cell cycle checkpoints, whereas those that BRCA1 inhibits are for cell division promoting genes. This is consistent with BRCA1 regulating checkpoints and cell proliferation. Particularly interesting in this context is the report that overexpression of BRCA1 can inhibit oestrogen receptor signalling $\left[25^{\circ}\right]$. This suggests a model whereby loss of BRCA1 could lead to an increased proliferative capacity of the breast epithelium in response to oestrogen. However, this appears to be at odds with clinical findings that show loss of the oestrogen receptor in a high proportion of BRCA-associated breast tumours [33,34].

A major problem with many of the studies described above is that overexpression of BRCA1 itself, especially at inappropriate phases of the cell cycle, could cause genotoxic stress and thus lead to expression of stress-induced genes as a secondary effect. Experiments using $B R C A$-null cells in which exogenous BRCA expression is induced to wild-type levels at the appropriate time in the cell cycle would rule out this possibility.

\section{DNA repair}

Mouse cells with Brca1 or Brca2 mutations are hypersensitive to ionizing radiation, a genotoxic treatment that causes primarily double-strand breaks in DNA [35 $\left.{ }^{\bullet-}-38^{\bullet}\right]$. This suggests that Brca1 and Brca2 play a part in the cellular response to DNA double-strand breaks. This function may be restricted to part of the cell cycle, however, because Brca 2 mutant cells can repair the double-strand breaks that arise during $\mathrm{V}(\mathrm{D}) \mathrm{J}$ recombination [38••] (Bertwistle D, Ashworth A, unpublished observations); these occur during the $G_{1}$ phase of the cell cycle, when nonhomologous end joining (NHEJ) is thought to repair most double-strand breaks [39]. Expression of BRCA1 and BRCA2 is very low during $G_{1}$ phase, but is induced as 
cells enter $S$ phase $\left[40^{\circ}, 41^{\circ}\right]$. During the late $S$ and $G_{2}$ phases of the cell cycle, when there are two copies of chromosomes, one copy can be used as a template to repair double-strand breaks in the other by homologous recombination [39]. In dividing mammalian cells as many as $30-50 \%$ of DNA double-strand breaks may be repaired this way [42]. RAD51, a protein that plays a key role in homologous recombination, has been shown to associate with both BRCA1 and BRCA2 $\left[8^{\bullet}, 9^{\bullet}, 35^{\bullet \bullet}, 43,44^{\bullet \bullet}\right]$. Furthermore, BRCA1 also associates with the RAD50-MRE11-nibrin complex, which is thought to process DNA double-strand breaks for repair by both NHEJ and homologous recombination [45 $\left.{ }^{\circ}\right]$. Together, these data suggest that BRCA1 and BRCA2 are involved in homologous recombination-mediated repair of DNA double-strand breaks. To complicate matters, however, there is also some evidence that BRCA1 may have a role in the mechanistically independent process of the transcription-coupled repair of oxidative DNA damage [46].

Spontaneous chromosomal abnormalities are observed at high frequency in untreated Brcal and Brca2 mutant cells, implying that these genes act to repair DNA damage that occurs as a consequence of normal cell division, as well as that caused by genotoxic agents $\left[36^{\bullet \bullet}, 38^{\bullet \bullet}\right.$. The nature of the chromosomal aberrations suggests a defect in the repair of DNA damage sustained during the $S$ or $G_{2}$ phase of the cell cycle. Because BRCA1 and BRCA2 are associated with RAD51, it is significant that loss of RAD51 causes chicken DT40 cells to spontaneously accumulate DNA double-strand breaks, arrest at the $\mathrm{G}_{2} / \mathrm{M}$ phase transition and die [47]. A possible explanation for such severe phenotypes comes from studies in bacteria, which suggest that double-strand breaks occur frequently as a normal consequence of DNA replication and are repaired by homologous recombination [48]. The role of homologous recombination in DNA replication in eukaryotes is less clear. Holliday junction recombination intermediates do spontaneously arise in normal yeast cells during $S$ phase, however [49]. This suggests that homologous recombination repairs DNA damage that occurs spontaneously during DNA replication in eukaryotic as well as in prokaryotic cells. The embryonic lethality observed in Rad51 null mice [50] and many Brca1 and Brca2 mutant mouse strains may therefore be due to a failure of DNA replication.

\section{Cell cycle checkpoint activation and loss}

Cells respond to DNA damage by activating checkpoints that prevent their progression through the cell cycle. The spontaneous DNA damage observed in Brca1 and Brca2 mutant cells would be predicted to activate such checkpoints. Indeed, these cells suffer from a proliferative defect, probably due to checkpoint activation caused by upregulation of the CDK inhibitor p21 ${ }^{\mathrm{WAF}} 1\left[29^{\bullet \bullet}, 37^{\bullet \bullet}\right.$, p53, which is upregulated in Brca2 mutant cells. Therefore, loss of the p53 pathway would be predicted to alleviate the proliferation defect observed in Brca1 and Brca2 mutant cells. Although this may be the case for some mutations $\left[51^{\bullet \bullet}\right.$, others are only partially rescued [52 ${ }^{\bullet}$ ] (Connor $\mathrm{F}$, Ashworth A, unpublished observation), suggesting that this is not the only checkpoint that is activated. Nevertheless, loss of the p53 pathway appears to be important for the progression of $B R C A$-associated tumours. Supporting this hypothesis is the finding that $p 53$ mutations are found at a higher frequency in $B R C A$-associated breast tumours than in sporadic breast tumours $\left[53^{\bullet}, 54^{\bullet \bullet}\right]$.

Checkpoints exist at $G_{1} / S, S, G_{2} / M$ and $M$ in the cell cycle, and all these appear to be operational in Brca2 mutant cells $\left[38^{\bullet \bullet}, 51^{\bullet \bullet}\right.$. In contrast, cells in which exon 11 of Brca 1 is deleted have an intact $\mathrm{G}_{1} / \mathrm{S}$ checkpoint, but are defective in an ionizing radiation-induced $\mathrm{G}_{2} / \mathrm{M}$ checkpoint $\left[55^{\circ}\right.$ ]. Furthermore, cells overexpressing a C-terminal fragment of BRCA1 fail to arrest when treated with the spindle inhibitor colchicine [56]. These results suggest that BRCA1 may also be involved in the $\mathrm{G}_{2} / \mathrm{M}$ and spindle checkpoints.

The spindle checkpoint acts to monitor the fidelity of chromosome segregation during mitosis [57]. Chromosome segregation is controlled by the mitotic spindle, a system of microtubules organized by the centrosome. During mitosis, there are two centrosomes, one at either end of the mitotic spindle, and one diploid set of chromosomes segregates to each. The spindle checkpoint prevents chromosome missegregation by ensuring that each chromosome pair has recruited microtubules from both poles of a bipolar spindle, before chromosome segregation occurs at anaphase.

At the end of mitosis each daughter cell inherits one of the two centrosomes, and duplicates this at the $G_{1} / S$ phase transition so that it has two centrosomes during mitosis [58]. Recent studies have found that a high proportion of Brca1 and Brca2 mutant cells contain supernumerary centrosomes $\left[55^{\bullet}, 59^{\bullet}\right]$. There is thought to be no checkpoint monitoring the number of centrosomes in a cell, and therefore cells with excess centrosomes can enter mitosis [60]. Some of these cells might be predicted to arrest during mitosis due to the activation of the spindle checkpoint, however. Indeed, the growth of Brca2 mutant cells can be rescued by the expression of a dominant negative mutant of the spindle checkpoint gene BUB1 [51*]. This implies that some Brca2 mutant cells activate the spindle checkpoint.

Under certain circumstances, however, mitoses with supernumerary centrosomes can fail to activate the spindle checkpoint [60] and this could lead to chromosome missegregation [61]. The consequence of this would be chromosome gain or loss and therefore aneuploidy, which is 
observed in a large proportion of Brca1 and Brca2 mutant cells $\left[55^{\bullet}, 59^{\bullet \bullet}\right]$. These results suggest that Brca1 and Brca2 may regulate centrosome duplication. The finding that BRCA1 associates with centrosomal proteins supports this hypothesis [62]. Alternatively, the excess of centrosomes in Brca1 and Brca2 mutant cells may be a secondary effect; centrosomes can undergo multiple rounds of duplication during $S$-phase arrest leading to supernumerary centrosomes $[60,63]$. Whatever the mechanism of centrosome amplification in Brca1 and Brca2 mutant cells, this phenomenon suggests that mutations in BRCA1 and BRCA2 might cause chromosomal instability in human tumours. It will be interesting to know whether these tumours have a high frequency of mutations in spindle checkpoint genes.

The chromosomal aberrations and aneuploidy found in mouse cells mutant for Brca1 or Brca2 are in agreement with the pathological data showing chromosomal instability in $B R C A$-associated breast tumours [64. $4^{\circ}$. In the light of these profound and irreversible changes, therapeutic strategies involving the reintroduction of $B R C A 1$ or $B R C A 2$ into tumours [65] may well be viewed as closing the stable door after the horse has bolted.

\section{Conclusion}

Our understanding of the precise functions of the BRCA1 and BRCA2 proteins and how their loss leads to tumourigenesis is still incomplete. However, we now have several clues as to the general cellular processes in which these proteins are involved, and this understanding has already illuminated some of the pathological features of $B R C A$ associated breast tumours. Activation or repression of transcriptional targets of the BRCA1 protein might play a role in tumour initiation or progression. These changes in gene expression may underpin some of the pathological changes associated with the tumours. Lack of activation of stressinduced checkpoint genes could contribute to aneuploidy. Failure to inhibit growth promoting genes could increase and disregulate proliferation. The genetic instability observed in mouse models and human tumours most likely reflects a role for BRCA1 and BRCA2 in homologous recombination and possibly in centrosome regulation. This effect may manifest itself relatively early in breast cancer progression, increasing the probability of tumourigenesis rather than directly promoting tumour growth. The synergy between mutations in $p 53$ and the $B R C A$ genes in tumours indicates a role for loss of checkpoint control in tumour progression. Taken together these findings give an insight into the pathogenesis of $B R C A$-associated tumours and will inform future therapeutic strategies.

\section{References}

Articles of particular interest have been highlighted as:

- of special interest

- of outstanding interest

1. Rahman N, Stratton MR: The genetics of breast cancer susceptibility. Annu Rev Genet 1998, 32:95-121.
2. Bertwistle D, Ashworth A: Functions of the BRCA1 and BRCA2 genes. Curr Opin Genet Dev 1998, 8:14-20.

3. Easton D: Breast cancer genes: what are the real risks? Nature Genet 1997, 16:210-211.

4. Miki Y, Swensen J, Shattuck-Eidens D, et al: A strong candidate for - the breast and ovarian cancer susceptibility gene BRCA1. Science 1994, 266:66-71.

Identification of the first hereditary breast cancer gene, BRCA1.

5. Wooster R, Bignell G, Lancaster J, et al: Identification of the breast - $\quad$ cancer susceptibility gene BRCA2. Nature 1995, 378:789-791. First report of the identification of the second hereditary breast cancer gene, BRCA2.

6. Bork P, Blomberg N, Nilges $\mathrm{M}$ : Internal repeats in the BRCA2 - $\quad$ protein sequence. Nature Genet 1996, 13:22-23.

See annotation for [9*].

7. Bignell G, Micklem G, Stratton MR, Ashworth A, Wooster R: The BRC

- repeats are conserved in mammalian BRCA2 proteins. Hum Mol Genet 1997, 6:53-58.

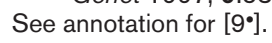

8. Chen PL, Chen CF, Chen Y, et al: The BRC repeats in BRCA2 are - critical for RAD51 binding and resistance to methyl methanesulfonate treatment. Proc Natl Acad Sci USA 1998, 95:5287-5292. See annotation for $\left[9^{\circ}\right]$.

9. Wong AKC, Pero R, Ormonde PA, Tavtigian SV, Bartel PL: RAD51 - interacts with the evolutionarily conserved BRC motifs in the human breast cancer susceptibility gene BRC. J Biol Chem 1997, 272:31941-31944.

Bork et al [6.] identified eight repeats of 30-80 amino acids each in part of the BRCA2 protein encoded by exon 11. In the paper by Bignell et al [7'], comparison of the BRCA2 exon 11 sequence from six mammalian species reveals that, unlike the rest of exon 11, these 'BRC' repeats are highly conserved, suggesting that they are important for the functionality of BRCA2. Both the paper by Chen et al [ $\left.8^{\circ}\right]$ and this paper demonstrate that the BRC repeats bind RAD51.

10. Breast Cancer Linkage Consortium: Pathology of familial breast - cancer: differences between breast cancers in carriers of BRCA1 or BRCA2 mutations and sporadic cases. Lancet 1997, 349: $1505-1510$.

Comparison of sporadic tumours with those from BRCA1 and BRCA2 mutation carriers. Differences are observed in the pathology of the three groups, implying that tumourigenesis may proceed by distinct mechansims in the three types of tumour.

11. Chapman MS, Verma IM: Transcriptional activation by BRCA1. - Nature 1996, 382:678-679.

See annotation for [13०].

12. Monteiro ANA, August $\mathrm{A}$, Hanafusa $\mathrm{H}$ : Evidence for a transcriptional - activation function of BRCA1 C-terminal region. Proc Natl Acad Sci USA 1996, 93:13595-13599.

See annotation for [13*].

13. Haile DT, Parvin JD: Activation of transcription in vitro by the - BRCA1 carboxyl-terminal domain. J Biol Chem 1999, 274: 2113-2117.

This paper, along with $\left[11^{\bullet}, 12^{\circ}\right]$, demonstrate that the C-terminal region of BRCA1 can act as a transcriptional activator, when fused to a heterologous DNA-binding domain. These results suggest that BRCA1 may act to regulate transcription.

14. Scully R, Anderson SF, Chao DM, et al: BRCA1 is a component of - the RNA polymerase II holoenzyme. Proc Natl Acad Sci USA 1997, 94:5605-5610.

Endogenous BRCA1 is found to copurify with the RNA polymerase II holoenzyme over multiple chromatographic steps, supporting a role for BRCA1 in transcriptional regulation.

15. Anderson SF, Schlegel BP, Nakajima T, Wolpin ES, Parvin JD: BRCA1 protein is linked to the RNA polymerase II holoenzyme complex via RNA helicase A. Nature Genet 1998, 19:254-256.

16. Neish AS, Anderson SF, Schlegel BP, Wei W, Parvin JD: Factors associated with the mammalian RNA polymerase II holoenzyme. Nucleic Acids Res 1998, 26:847-853. 
17. Maldonado E, Shiekhattar R, Sheldon M, et al: A human RNA polymerase II complex associated with SRB and DNA-repair proteins. Nature 1996, 381:86-89.

18. Niculescu AB III, Chen X, Smeets M, et al: Effects of p21(Cip1/Waf1) at both the G1/S and the G2/M cell cycle transitions: pRb is a critical determinant in blocking DNA replication and in preventing endoreduplication. Mol Cell Biol 1998, 18:629-643.

19. Somasundaram $\mathrm{K}$, Zhang $\mathrm{H}$, Zeng $\mathrm{YX}$, et al: Arrest of the cell cycle - by the tumour-suppressor BRCA1 requires the CDK-inhibitor p21 WAF1. Nature 1997, 389:187-190.

Overexpression of BRCA1 is shown to induce expression of the endogenous p21WAF1 protein and arrest the cell cycle in p21WAF1+l+, but not p2 $1_{\text {WAF1-I- }}$ cells. Given the proposed role for BRCA1 in transcriptional regulation, this suggests that the p21WAF1 gene is a target for regulation by BRCA1.

20. Zhang $\mathrm{H}$, Somasundaram $\mathrm{K}$, Peng $\mathrm{Y}$, et al: BRCA1 physically associates with p53 and stimulates its transcriptional activity. Oncogene 1998, 16:1713-1721.

21. Ouchi T, Monteiro AN, August A, Aaronson SA, Hanafusa H: BRCA1 regulates p53-dependent gene expression. Proc Natl Acad Sci USA 1998, 95:2302-2306.

22. Chai YL, Cui J, Shao N, et al: The second BRCT domain of BRCA1 proteins interacts with p53 and stimulates transcription from the p21WAF1/CIP1 promoter. Oncogene 1999, 18:263-268.

23. Harkin DP, Bean JM, Miklos D, et al: Induction of GADD45 and - JNK/SAPK-dependent apoptosis following inducible expression of BRCA1. Cell 1999, 97:575-586.

Overexpression of BRCA1 is shown to induce expression of the endogenous GADD45 gene. Given the proposed role for BRCA1 in transcriptional regulation, this suggests that GADD45 is a target for regulation by BRCA1.

24. Wang Q, Zhang H, Kajino K, Greene MI: BRCA1 binds c-Myc and inhibits its transcriptional and transforming activity in cells. Oncogene 1998, 17:1939-1948.

25. Fan S, Wang J, Yuan R, et al: BRCA1 inhibition of estrogen recep- $\quad$ tor signaling in transfected cells. Science 1999, 284:1354-1356. Activation of an oestrogen receptor-responsive promoter construct by the oestrogen receptor is shown to be inhibited by overexpression of BRCA1. This suggests a role for BRCA1 in modulating oestrogen receptor signalling.

26. Yu X, Wu LC, Bowcock AM, Aronheim A, Baer R: The C-terminal

- (BRCT) domains of BRCA1 interact in vivo with CtIP, a protein implicated in the CtBP pathway of transcriptional repression. J Biol Chem 1998, 273:25388-25392.

See annotation for $\left[2^{\circ}\right]$.

27. Li S, Chen PL, Subramanian T, et al: Binding of CtIP to the BRCT - repeats of BRCA1 involved in the transcription regulation of p21 is disrupted upon DNA damage. J Biol Chem 1999, 274 :11334-11338. This paper and $\left[2^{\circ}\right]$ demonstrate that BRCA1 interacts with CtIP, which binds to the transcriptional corepressor CtBP. This suggests a mechanism for transcriptional regulation by BRCA1. The interaction is disrupted upon DNA damage, implying that DNA damage modulates the proposed transcriptional regulatory activity of BRCA1.

28. Yarden RI, Brody LC: BRCA1 interacts with components of the - histone deacetylase complex. Proc Natl Acad Sci USA 1999, 96: 4983-1988.

Demonstrates that BRCA1 can interact with parts of the histone deactylase complex. This is consistent with the proposed roles for BRCA1 in transcriptional regulation and DNA repair, both processes being likely to require chromatin remodelling.

29. Hakem R, de la Pompa JL, Sirard C, et al: The tumor suppressor -. gene Brca1 is required for embryonic cellular proliferation in the mouse. Cell 1996, 85:1009-1023.

Description of the phenotypes of $\mathrm{Brca} 1^{+/-}$and $\mathrm{Brca} 1^{-/-}$mice. Whereas heterozygotes appear normal, homozygotes die during early embryogenesis. Embryonic lethality may be due to reduced cell proliferation, which is accompanied by a dramatic increase in the level of $p 21^{\text {WAF1 }}$ gene expression.
30. Milner J, Ponder B, Hughes-Davies L, Seltmann M, Kouzarides T: - Transcriptional activation functions in BRCA2. Nature 1997, 386: 772-773.

Parts of BRCA2 encoded by exon 3 can act as a transcriptional activator when fused to a heterologous DNA-binding domain. This suggests that BRCA2 may act to regulate transcription.

31. Siddique H, Zou JP, Rao VN, Reddy ES: The BRCA2 is a histone - acetyltransferase. Oncogene 1998, 16:2283-2285.

See annotation for [32]

32. Fuks F, Milner J, Kouzarides T: BRCA2 associates with acetyltrans- ferase activity when bound to P/CAF. Oncogene 1998, 17 2531-2534.

This paper and $\left[31^{\circ}\right]$ report that BRCA2 is associated with HAT activity. Evidence that BRCA2 itself possesses HAT activity was presented by Sid

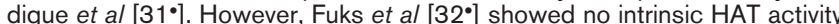
for BRCA2, and argued that BRCA2 is associated with HAT activity due to an interaction with the transcriptional coactivator P/CAF.

33. Robson $\mathrm{M}$, Rajan $\mathrm{P}$, Rosen $\mathrm{PP}$, et al: BRCA-associated breast cancer: absence of a characteristic immunophenotype. Cancer Res 1998, 58:1839-1842.

34. Osin P, Crook T, Powles T, Peto J, Gusterson B: Hormone status of in-situ cancer in BRCA1 and BRCA2 mutation carriers. Lancet 1998, 351:1487.

35. Sharan SK, Morimatsu M, Albrecht U, et al: Embryonic lethality and -. radiation hypersensitivity mediated by Rad51 in mice lacking Brca2. Nature 1997, 386:804-810.

Description of the phenotypes of $B r c a 2^{+/-}$and $B r c a 1^{-1-}$ mice. Whereas heterozygotes appear normal, homozygotes die during early embryogenesis. A role for Brca2 in DNA repair is suggested by data showing that Brca2 and Rad51 interact and that $\mathrm{Brca2}^{-/-}$embryos are hypersensitive to gamma irradiation.

36. Shen SX, Weaver $\mathrm{Z}, \mathrm{Xu} X$, et al: $\mathrm{A}$ targeted disruption of the -. murine Brca1 gene causes gamma-irradiation hypersensitivity and genetic instability. Oncogene 1998, 17:3115-3124.

Brca1 homozygous mutant mouse embryos are shown to be hypersensitive to gamma-irradiation, suggesting a role for Brca1 in DNA repair. Analysis of metaphase chromosomes from the mutant embryos reveals numerical and structural aberrations, which occur at even higher frequencies on a p53 mutant background. Neither Brca1 $1^{-1-}$, nor Brca $1^{-1-} p 53^{-1-}$ fibroblasts are viable, but proliferative foci arise from $B r c a 1^{-1-} p 53^{-1-}$ cells, suggesting that mutations in Brca1 and p53 cooperate in tumourigenesis.

37. Connor F, Bertwistle D, Mee PJ, et al: Tumorigenesis and a DNA -• repair defect in mice with a truncating Brca2 mutation. Nature Genet 1997, 17:423-430

See annotation for [38**].

38. Patel KJ, Vu VP, Lee $\mathrm{H}$, et al: Involvement of Brca2 in DNA repair. -. Mol Cell 1998, 1:347-357.

Previously reported mutations of the Brca2 gene $\left[35^{\bullet *}, 52^{\bullet \bullet}\right]$ were lethal early in embryogenesis when homozygous. This paper and $\left[37^{\circ *}\right]$ report the generation of viable Brca2 mutant mouse strains that survive to develop thymic lymphomas. Fibroblasts from Brca2 mutant embryos are shown to have a proliferative defect accompanied by upregulation of p53 and p21WAF1. This may reflect the activation of DNA damage checkpoints. The demonstration of a defect in DNA repair [37.*] and a high frequency of

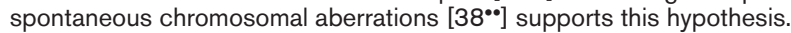

39. Takata M, Sasaki MS, Sonoda E, et al: Homologous recombination and non-homologous end-joining pathways of DNA double-strand break repair have overlapping roles in the maintenance of chromosomal integrity in vertebrate cells. $E M B O J 1998$, 17:5497-508.

40. Chen Y, Farmer AA, Chen CF, et al: BRCA1 is a 220-kDa nuclear - phosphoprotein that is expressed and phosphorylated in a cell cycle-dependent manner. Cancer Res 1996, 56:3168-3172.

See annotation for $\left[41^{\circ}\right]$.

41. Bertwistle D, Swift S, Marston NJ, et al: Nuclear location and cell - cycle regulation of the BRCA2 protein. Cancer Res 1997, 57: 5485-5488.

This study and [40 ${ }^{\circ}$ report that expression of BRCA1 and BRCA2 are regulated through the cell cycle. Both proteins are present at low levels during $G_{1}$ phase and are induced as cells enter the cell cycle at $G_{1} / S$. 
42. Liang $\mathrm{F}$, Han M, Romanienko PJ, Jasin M: Homology-directed repair is a major double-strand break repair pathway in mammalian cells. Proc Natl Acad Sci USA 1998, 95:5172-5177.

43. Baumann $\mathrm{P}$, West SC: Role of the human Rad51 protein in homologous recombination and double-strand break repair. Trends Biochem Sci 1998, 23:247-251.

44. Scully R, Chen J, Plug A, et al: Association of BRCA1 with Rad51 in - $\quad$ mitotic and meiotic cells. Cell 1997, 88:265-275.

This paper demonstrates colocalization of BRCA1 and RAD51 in nuclear foci during $S$ phase in mitotic cells and on unsynapsed elements of the synaptonemal complex in meiotic cells. Moreover, the two proteins are shown to associate by co-immunoprecipitation. This association was the first data to suggest a role for BRCA1 in DNA repair and recombination.

45. Zhong $\mathrm{Q}$, Chen CF, Li S, et al: Association of BRCA1 with the - hRad50-hMre11-p95 complex and the DNA damage response. Science 1999, 285:747-750.

The Rad50-MRE11-p95-nibrin complex is involved in both NHEJ and homologous recombination-mediated repair of DNA double-strand breaks. This paper demonstrates that a proportion of BRCA1 is associated with this complex, and that BRCA1 is required for the irradiation induced formation of the complex.

46. Gowen LC, Avrutskaya AV, Latour AM, Koller BH, Leadon SA: BRCA1 required for transcription-coupled repair of oxidative DNA damage. Science 1998, 281:1009-1012.

47. Sonoda E, Sasaki MS, Buerstedde JM, et al: Rad51-deficient vertebrate cells accumulate chromosomal breaks prior to cell death EMBO J 1998, 17:598-608.

48. Michel B, Ehrlich SD, Uzest M: DNA double-strand breaks caused by replication arrest. EMBO J 1997, 16:430-438.

49. Zou H, Rothstein R: Holliday junctions accumulate in replication mutants via a RecA homolog-independent mechanism. Cell 1997 90:87-96

50. Lim DS, Hasty P: A mutation in mouse Rad51 results in an early embryonic lethal that is suppressed by a mutation in $p 53 . \mathrm{Mol} \mathrm{Cell}$ Biol 1996, 16:7133-7143.

51. Lee $\mathrm{H}$, Trainer $\mathrm{AH}$, Friedman LS, et al: Mitotic checkpoint inactiva-. tion fosters transformation in cells lacking the breast cancer susceptibility gene, Brca2. Mol Cell 1999, 4:1-10.

Loss of BRCA2 is tumourigenic. Paradoxically, fibroblasts from embryos homozygous for $\mathrm{Brca} 2$ mutations suffer from a proliferative arrest $\left[37 \cdot \bullet, 38 \bullet^{\bullet}\right]$. This paper demonstrates that inactivation of checkpoints responsive to mitotic spindle disruption, by mutant forms of p53 or Bub1, relieves this growth arrest. Therefore, loss-of-function mutations in mitotic checkpoint genes may cooperate with BRCA2 mutation in inherited breast cancer.

52. Ludwig T, Chapman DL, Papaioannou VE, Efstratiadis A: Targeted

- mutations of breast cancer susceptibility gene homologs in mice: lethal phenotypes of Brca1, Brca2, Brca1/Brca2, Brca1/p53 and Brca2/p53 nullizygous embryos. Genes Dev 1997, 11:1226-1241. This study describes mutations in Brca1 and Brca2 that have an embryonic lethal phenotype when homozygous. Both mutations are partially rescued on a p53 mutant background, with embryos surviving slightly longer.

53. Crook T, Crossland S, Crompton MR, Osin P, Gusterson BA: p53 -• mutations in BRCA1-associated familial breast cancer. Lancet 1997, 350:638-639.

See annotation for $\left[54^{\bullet *}\right]$.

54. Crook T, Brooks LA, Crossland S, et al: p53 mutation with frequent -. novel condons but not a mutator phenotype in BRCA1- and BRCA2-associated breast tumours. Oncogene 1998, 17:1681-1689. In [53*0] the frequency of $p 53$ mutation is found to be higher in BRCA1. associated than in sporadic breast cancers. This study extends this finding to BRCA2-associated breast cancers.

55. Xu X, Weaver Z, Linke SP, et al: Centrosome amplification and a -• defective G2-M cell cycle checkpoint induce genetic instability in BRCA1 exon 11 isoform-deficient cells. Mol Cell 1999, 3:389-395. This paper analyzes mouse embryonic fibroblasts with a targeted deletion of exon 11 of the Brca1 gene. The mutant cells are shown to have a defective $\mathrm{G}_{2} / \mathrm{M}$ checkpoint, and as many as $25 \%$ have an excess of centrosomes. Both of these abnormalities are likely to lead to genetic instability.
56. Larson JS, Tonkinson JL, Lai MT: A BRCA1 mutant alters G2-M cell cycle control in human mammary epithelial cells. Cancer Res 1997, 57:3351-3355.

57. Hardwick KG: The spindle checkpoint. Trends Genet 1998, 14:1-4

58. Zimmerman W, Sparks CA, Doxsey SJ: Amorphous no longer: the centrosome comes into focus. Curr Opin Cell Biol 1999, $11: 122-128$

59. Tutt A, Gabriel A, Bertwistle D, et al: Absence of BRCA2 causes - genome instability by chromosome breakage and loss associated with centrosome amplification. Curr Biol 1999, 9:1107-1110.

Mouse embryonic fibroblasts homozygous for a mutation in Brca2 are shown to exhibit aneuploidy. An excess of centrosomes is also observed in these cells. These results suggest that loss of Brca2 causes aneuploidy by means of chromosome missegregation.

60. Sluder G, Thompson EA, Miller FJ, Hayes J, Rieder CL: The checkpoint control for anaphase onset does not monitor excess numbers of spindle poles or bipolar spindle symmetry. J Cell Sci 1997, 110:421-429.

61. Doxsey S: The centrosome: a tiny organelle with big potential. Nature Genet 1998, 20:104-106.

62. Hsu LC, White RL: BRCA1 is associated with the centrosome during mitosis. Proc Natl Acad Sci USA 1998, 95:12983-12988.

63. Hinchcliffe EH, Li C, Thompson EA, Maller JL, Sluder G: Requirement of Cdk2-cyclin $\mathrm{E}$ activity for repeated centrosome reproduction in Xenopus egg extracts. Science 1999, 283:851-854

64. Tirkkonen $\mathrm{M}$, Johannsson $\mathrm{O}$, Agnarsson $\mathrm{BA}$, et al: Distinct somatic - genetic changes associated with tumor progression in carriers of BRCA1 and BRCA2 germ-line mutations. Cancer Res 1997, 57: 1222-1227.

A genome-wide survey by comparative genome hybridization of breast cancers from $B R C A$-associated and control breast cancers. Tumours from $B R C A 1$ and $B R C A 2$ mutation carriers are found to have twice the number of genetic changes observed in control tumours. Furthermore, distinct genetic changes are seen in BRCA1, BRCA2 and control breast cancers.

65 Holt JT: Breast cancer genes: therapeutic strategies. Ann N Y Acad Sci 1997, 833:34-41

Author address: The Breakthrough Toby Robins Breast Cancer Research Centre, The Institute of Cancer Research, London, UK

Correspondence: David Bertwistle, The Breakthrough Toby Robins Breast Cancer Research Centre, The Institute of Cancer Research Fulham Road, London SW3 6JB, UK. Tel: +44 171352 8133; fax: +44 171352 3299; e-mail: davidb@icr.ac.uk 